\title{
LOCALIZATION OF DIFFUSIVE SOURCES USING SPATIOTEMPORAL MEASUREMENTS
}

\author{
Yue M. Lu ${ }^{1}$, Pier Luigi Dragotti ${ }^{2}$ and Martin Vetterli ${ }^{3}$ \\ ${ }^{1}$ Harvard School of Engineering and Applied Sciences, Cambridge, MA, USA \\ ${ }^{2}$ Imperial College London, UK \\ ${ }^{3}$ Ecole Polytechnique Fédérale de Lausanne (EPFL), Switzerland \\ Emails: yuelu@seas.harvard.edu; p.dragotti@imperial.ac.uk; martin.vetterli@epfl.ch
}

\begin{abstract}
Consider a diffusion field induced by a finite number of localized and instantaneous sources. In this paper, we study the problem of estimating these sources from the spatiotemporal samples taken by a network of spatially distributed sensors. We start by considering the case of a single source but with unknown activation time. We show that the diffusion field at any spatial location is a scaled and shifted version of a common prototype function, and that this function is the unique solution to a particular differential equation. This observation leads to an efficient algorithm that can estimate the unknown parameters of the source by solving a system of linear equations. Following this algorithm we then present an acquisition strategy based around the Strang-Fix condition to obtain the appropriate measurements from the sensors. We then extend this result to the case of multiple sources with different activation time. For the algorithm proposed in this work, the minimum number of sensors required is $d+1$, where $d$ is the spatial dimension of the field. This requirement is independent of the number of active sources.
\end{abstract}

Index Terms - diffusion field, source localization, finite rate of innovation, spatiotemporal sampling

\section{INTRODUCTION}

Diffusion models many important physical, biological and social phenomena, including temperature variations, air pollution dispersion, biochemical substance release, and epidemic dynamics. Sampling and reconstructing diffusion processes using a network of sensors have applications ranging from data center temperature monitoring [2] (detecting cold and hot spots responsible for energy inefficiencies) to environmental monitoring [1] and homeland security [6].

At the microscopic scale, diffusion describes the random motion of a large number of particles, migrating from regions of high concentration to those of low concentration. At the macroscopic scale, the "average" statistical distributions of

P. L. Dragotti was supported in part by a Global Research Award from the Royal Academy of Engineering.

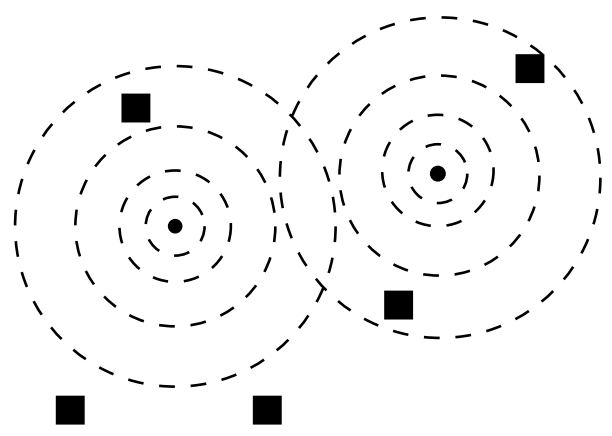

Fig. 1. Localization of point-like sources (shown as black dots) in a 2-D diffusive field based on the spatiotemporal samples taken by a network of sensors (shown as squares). Dashed lines represent the isolines of the diffusion fields induced by the two sources, respectively.

these particles are governed by the diffusion equation, originally derived by Fick [7] in 1855. In its simplest form-when the underlying medium is isotropic - the diffusion equation can be written as

$$
\frac{\partial}{\partial t} f(\boldsymbol{x}, t)=\mu \nabla^{2} f(\boldsymbol{x}, t)+s(\boldsymbol{x}, t),
$$

where $f(\boldsymbol{x}, t), \boldsymbol{x} \in \mathbb{R}^{d}$ is the spatiotemporal distribution of the field, $\mu$ is the diffusion coefficient, $\nabla^{2} f=\sum_{i=1}^{d} \frac{\partial^{2} f}{\partial x_{i}^{2}}$ is the (spatial) Laplacian of $f(\boldsymbol{x}, t)$, and $s(\boldsymbol{x}, t)$ represents the sources of the field.

In this paper, we consider the case where the unknown sources $s(x, t)$ are localized and instantaneous, i.e.,

$$
s(\boldsymbol{x}, t)=\sum_{k=1}^{K} c_{k} \delta\left(\boldsymbol{x}-\boldsymbol{x}_{k}, t-t_{k}\right),
$$

for some unknown parameters $\left\{c_{k}, \boldsymbol{x}_{k}, t_{k}\right\}_{k=1}^{K}$. In practice, this source model can describe sudden events (e.g., explosions or accidental release of pollutants) that are the key targets of various environmental monitoring or security applications.

As shown in Figure 1, we use a network of spatially distributed sensors to monitor the field. A sensor network can 
be seen as a sampling device that takes spatio-temporal measurements of the underlying phenomenon. However, it is also worth to highlight immediately the main differences between traditional sampling and sampling with sensor networks. In the traditional acquisition process, one measures the source of interest directly and in many cases the acquisition device which performs the filtering can be modified and designed to achieve the signal reconstruction. In a sensor network instead one does not have direct access to the source which is first filtered by the PDE that characterizes the field of interest (i.e., the diffusion equation in our scenario). Moreover in sensor networks, the sampling in time is different to the sampling in space for various reasons. First of all, sampling in time is normally uniform whereas the sampling in space is not since sensors are arbitrarily located. Secondly, in a sensor network it is possible to perform filtering in time but this is not the case for space. Finally, it is easier to increase the sampling density in time than in space the latter requiring the physical deployment of more sensors. For this last reason, a nice property of the algorithm presented in this paper is that the minimum number of sensors required to recover the sources is always going to be $d+1$ and this independently of the number of active sources in the field.

More specifically, in this paper, we start by considering the case where there is a single source in the field, but the activation time of the source is unknown. We show that the induced diffusion field at each sensor location can be written as a scaled and shifted version of a common prototype function. We further observe that this prototype function is the unique solution to a particular differential equation. This observation enables us to build a set of linear equations linking the unknown parameters (time instants and source-to-sensor distances) to local sensor measurements. Estimating the parameters of the source then reduces to solving a set of linear equations. Based on these results we propose an acquisition strategy that allows us to obtain the correct measurements from sensors. Finally we discuss how the proposed algorithm can be extended to the case of multiple source which are activated at different times.

In the rest of the paper, we assume that the field $f(\boldsymbol{x}, t)$ is supported on two spatial dimensions (i.e., $d=2$ ). However, our discussion and proposed algorithms can be easily extended to the general $d$-dimensional cases. This paper summarizes and extends results presented in [5].

\section{A PARAMETRIC FORM OF THE DIFFUSION FIELD}

We start by presenting a parametric form of the diffusion field governed by (1). Due to the linearity of (1), its solutions have the form

$$
f(\boldsymbol{x}, t)=(s * g)(\boldsymbol{x}, t),
$$

where $g(\boldsymbol{x}, t)$ is the Green's function of the equation and $*$ denotes convolutions along the spatial and temporal dimen- sions [4]. In this sense, the diffusion equation behaves exactly like a linear, shift-invariant system, linking the "input" $s(\boldsymbol{x}, t)$ to the induced "output" $f(\boldsymbol{x}, t)$ through a spatiotemporal impulse response $g(\boldsymbol{x}, t)$.

A closed-form expression for the Green's function is

$$
g(\boldsymbol{x}, t)=\frac{1}{4 \pi \mu t} e^{-\frac{\|\boldsymbol{x}\|^{2}}{4 \mu t}} U(t),
$$

where $\|\cdot\|$ denotes Euclidean norm and $U(t)$ is a unit-step function. On substituting (4) and (2) into (3), we get

$$
f(\boldsymbol{x}, t)=\sum_{k=1}^{K} \frac{c_{k}}{4 \pi \mu\left(t-t_{k}\right)} e^{-\frac{\left\|\boldsymbol{x}-\boldsymbol{x}_{k}\right\|^{2}}{4 \mu\left(t-t_{k}\right)}} U\left(t-t_{k}\right) .
$$

The diffusion field described above is not bandlimited in space or time; however, it is completely determined by a finite number of parameters, namely, the intensities, locations and activation time of the sources. For this reason, the induced field can be seen as a parametric signal with finite rate of innovation [9].

Assume now, that the field in (5) is observed with $N$ spatially-distributed sensors, located at $\left\{\boldsymbol{p}_{1}, \boldsymbol{p}_{2}, \ldots, \boldsymbol{p}_{N}\right\} \subset$ $\mathbb{R}^{2}$, respectively. For a fixed time $t$, the measurement $y_{n}(t)$ obtained with the $n$th sensor can be written as

$$
y_{n}(t)=\sum_{k=1}^{K} \frac{c_{k}}{4 \pi \mu\left(t-t_{k}\right)} e^{-\frac{D_{k, n}}{4 \mu\left(t-t_{k}\right)}} U\left(t-t_{k}\right),
$$

where

$$
D_{k, n} \stackrel{\text { def }}{=}\left\|\boldsymbol{x}_{k}-\boldsymbol{p}_{n}\right\|^{2}
$$

is the squared Euclidean distance between the $k$ th source and the $n$th sensor.

The estimation of both the positions and the activation time of the sources is in general difficult. In the next sections, we will first show how to estimate a single source with unknown activation time to then highlight extensions of this result to the case of multiple sources activated at different times.

\section{ESTIMATING A SINGLE SOURCE WITH UNKNOWN ACTIVATION TIME}

In this section, we start by considering the case when there is a single source in the field, but the activation time of the source is unknown. We then extend our discussion to the case of multiple independent sources.

\subsection{The Temporal Prototype Function of Diffusion Fields}

Our proposed algorithm is based on the following observation: at any spatial location, the diffusion field induced by sparse sources can be represented by a linear combination of a prototype function. To see this, we define

$$
\varphi(t) \stackrel{\text { def }}{=} \frac{1}{4 \pi t} e^{-1 /(4 t)} U(t)
$$




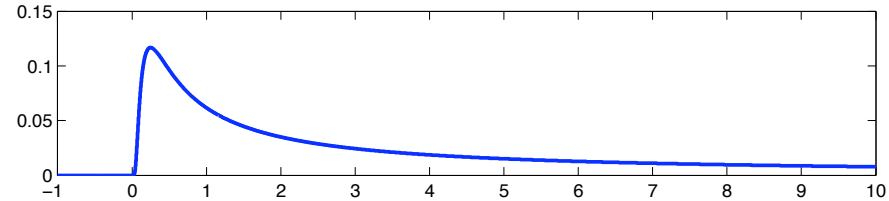

Fig. 2. The temporal prototype function $\varphi(t)$ of the diffusion process.

We can easily verify that, at any given spatial location $\boldsymbol{x}(\boldsymbol{x} \neq$ $\left.\boldsymbol{x}_{k}, 1 \leq k \leq K\right)$, the field $f(\boldsymbol{x}, t)$ in (5) can be written as

$$
f(\boldsymbol{x}, t)=\sum_{k=1}^{K} c_{k}^{\prime} \varphi\left(\frac{t-t_{k}}{s_{k}}\right)
$$

where $s_{k} \stackrel{\text { def }}{=}\left\|\boldsymbol{x}-\boldsymbol{x}_{k}\right\|^{2} / \mu$ and $c_{k}^{\prime} \stackrel{\text { def }}{=} c_{k} /\left(s_{k} \mu\right)$. In words, the diffusion field at any spatial location can be written as a linear combination of $K$ scaled and shifted versions of the prototype function $\varphi(t)$. Meanwhile, the scale and shift parameters correspond to the spatial locations and activation time of the sources, respectively.

In what follows, we present several important properties of the prototype function $\varphi(t)$. Figure 2 shows the values of $\varphi(t)$ over $t \in[-1,10]$. We can easily verify that $\varphi(t)$ reaches its maximum $\left(e^{-1} / \pi\right)$ at $t=1 / 4$, and that it decays at the rate of $1 / t$ for sufficiently large $t$.

A somewhat surprising fact is that, despite the existence of the unit step $U(t)$ in the definition (8), the function $\varphi(t)$ is smooth on the entire real line, i.e., it has derivatives of all orders over $t \in \mathbb{R}$.

Lemma 1 The function $\varphi(t)$ belongs to $C^{\infty}$ on the real line. Meanwhile, at $t=0$, the derivatives of $\varphi(t)$ of all orders are equal to zero.

Proof We prove by induction. Assume that $\varphi^{(k)}(0)=0$ for some $k \geq 0$. It is easy to verify that, for $t>0$,

$$
\varphi^{(k)}(t)=P\left(t^{-1}\right) e^{-1 /(4 t)},
$$

where $P\left(t^{-1}\right)$ is some polynomial of $t^{-1}$. It follows that

$$
\varphi^{(k+1)}(0) \stackrel{\text { def }}{=} \lim _{t \rightarrow 0^{+}}\left(P\left(t^{-1}\right) e^{-1 /(4 t)}-0\right) / t=0 .
$$

Lemma 2 The function $\varphi(t)$ satisfies the following differential equation:

$$
4 t^{2} \varphi^{\prime}(t)+(4 t-1) \varphi(t)=0, \quad \text { for } t \in \mathbb{R} .
$$

Proof This can be straightforwardly verified from the definition of $\varphi(t)$ in (8).

\subsection{Sampling and Reconstructing Diffusion Fields with One Localized Source}

Based on the properties of the prototype function $\varphi(t)$ presented above, we propose a sampling and reconstruction algorithm for diffusion fields induced by a single source $c \delta(\boldsymbol{x}-$ $\boldsymbol{\xi}, t-\tau)$ with unknown location and activation time.

Consider $y_{n}(t)$, the diffusion field observed at the $n$th sensor at location $\boldsymbol{p}_{n}$. It follows from (9) that

$$
y_{n}(t)=\frac{c}{D_{n}} \varphi\left(\frac{t-\tau}{D_{n} / \mu}\right),
$$

where $D_{n}=\left\|\boldsymbol{\xi}-\boldsymbol{p}_{n}\right\|^{2}$ is the squared distance between the source location $\boldsymbol{\xi}$ and the sensor location $\boldsymbol{p}_{n}$.

Proposition 1 The function $y_{n}(t)$ satisfies the following differential equation

$$
\begin{aligned}
\frac{y_{n}(t)}{4 \mu} D_{n}+\left(y_{n}(t)+2 t y_{n}^{\prime}(t)\right) & -y_{n}^{\prime}(t) \tau^{2} \\
& =t^{2} y_{n}^{\prime}(t)+t y_{n}(t),
\end{aligned}
$$

for $t \in \mathbb{R}$.

Proof This follows directly from Lemma 2. In particular, we can obtain (11) by replacing $t$ with $(t-\tau) \mu / D_{n}$ in (10) and rearranging the terms.

Consider the unknown parameters $\left[D_{n}, \tau, \tau^{2}\right]$ as an unknown vector in $\mathbb{R}^{3}$. Then, for each $t,(11)$ provides a different linear equation, whose coefficients can be obtained from $y_{n}(t)$ and $y_{n}^{\prime}(t)$. In practice, reliably estimating the derivative of a function is problematic, due to the noise amplification property of the differentiation operation. However, this concern for robustness can be alleviated by exploiting the linearity of (11), as shown in the following proposition.

Proposition 2 Let $w(t)$ be an arbitrary window function. Then

$$
\begin{aligned}
& \left(\int \frac{y_{n}(t) w(t)}{4 \mu} d t\right) D_{n}+\left(\int\left(y_{n}(t)+2 t y_{n}^{\prime}(t)\right) w(t) d t\right) \tau \\
& -\left(\int y_{n}^{\prime}(t) w(t) d t\right) \tau^{2}=\int\left(t^{2} y_{n}^{\prime}(t)+t y_{n}(t)\right) w(t) d t .
\end{aligned}
$$

Let $\left\{y_{n, m}\right\}_{m}$ denotes the set of measurements taken by the $n$th sensor. To apply the result of Proposition 2, we set the measurements to

$$
\begin{aligned}
& y_{n, 1+4 k}=\int \frac{y_{n}(t) w(t-k T)}{4 \mu} d t, \\
& y_{n, 2+4 k}=\int\left(y_{n}(t)+2 t y_{n}^{\prime}(t)\right) w(t-k T) d t, \\
& y_{n, 3+4 k}=-\int y_{n}^{\prime}(t) w(t-k T) d t, \\
& y_{n, 4+4 k}=\int\left(t^{2} y_{n}^{\prime}(t)+t y_{n}(t)\right) w(t-k T) d t,
\end{aligned}
$$


for $k=0,1$, and some fixed sampling interval $T>0$. In practice, the derivatives and integrations in (13) can be approximated by finite differences and finite sums on a dense sampling grid. An alternative strategy is discussed in Sec. 3.4 .

After obtaining the samples $y_{n, m}$, it follows from (12) that

$$
\left(\begin{array}{lll}
y_{n, 1} & y_{n, 2} & y_{n, 3} \\
y_{n, 5} & y_{n, 6} & y_{n, 7}
\end{array}\right)\left(\begin{array}{c}
D_{n} \\
\tau \\
\tau^{2}
\end{array}\right)=\left(\begin{array}{l}
y_{n, 4} \\
y_{n, 8}
\end{array}\right)
$$

Note that (14) gives two linear constraints for three variables. To solve for the unknown parameters uniquely, we can either take more temporal samples [by setting $k=2,3, \ldots$ in (13)] or incorporate measurements taken at different sensors. The latter approach is feasible because each additional sensor brings in two more linear equations in the form of (14) but only one new variable (i.e., $D_{n}$ ), while $\tau$ and $\tau^{2}$ remain the same for all sensors.

Finally, once we obtain $\left\{D_{n}\right\}$, i.e., the squared distances from the source to the sensors, the spatial location of the source is recovered with the technique presented in the next section. For that purpose, we will see that we need at least three different sensors.

\subsection{Source Localization from Distance Information}

Expanding (7), we have the following set of equations

$$
D_{k, n}=\left\langle\boldsymbol{x}_{k}-\boldsymbol{p}_{n}, \boldsymbol{x}_{k}-\boldsymbol{p}_{n}\right\rangle=\left\|\boldsymbol{x}_{k}\right\|^{2}-2 \boldsymbol{p}_{n}^{T} \boldsymbol{x}_{k}+\left\|\boldsymbol{p}_{n}\right\|^{2},
$$

where $\left\{D_{k, n}\right\}$ and $\left\{\boldsymbol{p}_{n}\right\}$ are known parameters, and $\left\{\boldsymbol{x}_{k}\right\}$ are the unknowns to be estimated. To be clear, (15) is a quadratic equation of $\boldsymbol{x}_{k}$. However, by treating $\left\{\left\|\boldsymbol{x}_{k}\right\|^{2}\right\}$ and $\left\{\boldsymbol{x}_{k}\right\}$ as if they were independent free variables, we can view (15) as a set of $N$ linear equations with 3 unknowns. When $N \geq$ $d+1=3$, we can obtain $\left\{\boldsymbol{x}_{k}\right\}$ by simply solving a system of linear equations.

\subsection{Sampling one localized source using Strang-Fix ker- nels}

In light of the previous analysis, we now propose a sampling scheme centered around kernels $\phi(t)$ satisfying the StrangFix conditions [8, 3]. Such kernels together with their shifted versions are able to reproduce polynomials up to a certain degree $P$ :

$$
\sum_{n} c_{n, k}^{(P)} \phi(t-n)=t^{k}, \quad k=0,1, \ldots, P
$$

for a proper choice of the coefficients $c_{n . k}^{(P)}$.

We propose that the field be measured using a twochannel device. The first channel multiplies in analogue the incoming signal with a periodic compact support function $w(t)$, while the second channel executes the same operation but using the derivative of $w(t)$. The two resulting signals are then filtered and sampled with two Strang-Fix kernels. Note that we assume for simplicity the same sampling kernel for both channels, however, this hypothesis can be relaxed. The sampling kernel is assumed to be able to reproduce polynomial of at least degree two. Therefore sensor $n$ measures the following samples (recall that the index $n$ is for the sensor, while $m$ is for the sampling in time):

$$
y_{n, m}=\left\langle y_{n}(t) w(t), \phi(t-m)\right\rangle
$$

and

$$
y_{n, m}^{\prime}=\left\langle y_{n}(t) w^{\prime}(t), \phi(t-m)\right\rangle .
$$

Given the samples we use the polynomial reproduction property to obtain the following:

$$
\lambda_{n, k}=\sum_{m} c_{m, k}^{(P)} y_{n, m}=\int_{0}^{T_{1}} t^{k} y_{n}(t) w(t) d t \quad k=0,1,
$$

Similarly, one can obtain

$$
\lambda_{n, k}^{\prime}=\int_{0}^{T_{1}} t^{k} y_{n}^{\prime}(t) w(t) d t \quad k=0,1,2 .
$$

Note that $T_{1}$ is the duration of the window $w(t)$. With this measurements we can then construct and solve the system of equations in (14).

\subsection{Multiple Sources with Different Activation Time}

The proposed technique cannot unfortunately be directly extended to the case of multiple sources since the differential equation in (11) is not satisfied when multiple sources are active simultaneously. However, if sources get activated sequentially and if the interval of time between two consecutive activations is sufficiently large then one can use the above method sequentially in that one can estimate the first active source and then remove the contribution of this source from the following measurements in order to estimate the following sources.

\section{CONCLUSION}

We studied the problem of estimating the unknown sources (including their intensities, spatial locations, and activation time) of a diffusion field from the spatiotemporal samples taken by a network of spatially distributed sensors. When there is a single source but with unknown activation time, we showed that the sensor measurements at any sensor location is a scaled and shifted version of a common prototype function. The properties of the prototype function leads to a set of linear constraints, linking the unknown parameters of the source to local sensor measurements. Estimating the spatial location and activation time of the pointwise sources then boils down to solving a set of linear equations. This reconstruction strategy has then been extended to the case of multiple sources with different activation times. 


\section{REFERENCES}

[1] G. Barrenetxea, F. Ingelrest, G. Schaefer, M. Vetterli, O. Couach, and M. Parlange. SensorScope: Out-of-the-box environmental monitoring. In Proc. 7th Int. Conf. on Information Processing in Sensor Networks (IPSN 2008), St. Louis, USA, Apr. 2008.

[2] R. Bianchini and R. Rajamony. Power and energy management for server systems. Computer, 37(11):68-76, November 2004.

[3] P.L. Dragotti, M. Vetterli, and T. Blu. Sampling moments and reconstructing signals of finite rate of innovation: Shannon meets Strang-Fix. IEEE Trans. Signal Process., 55(5):1741-1757, May 2007.

[4] L. C. Evans. Partial Differential Equations. American Mathematical Society, Providence, RI, 1998.

[5] Y. Lu, P.L. Dragotti, and M. Vetterli. Localizing point sources in diffusive fields from spatiotemporal samples. In Proc. of Int. Conf. on Sampling Theory and Applications (SampTA), Singapore, May 2011.

[6] A. Nehorai, B. Porat, and E. Paldi. Detection and localization of vapor-emitting sources. IEEE Trans. Signal Process., 43(1):243-253, Jan. 1995.

[7] J. Philibert. One and a half century of diffusion: Fick, Einstein, before and beyond. Diffusion Fundamentals, 2(1):1-10, 2005.

[8] G. Strang and G. Fix. A Fourier analysis of the finite element variational method. In Constructive Aspect of Functional Analysis, pages 796-830, Rome, Italy, 1971.

[9] M. Vetterli, P. Marziliano, and T. Blu. Sampling signals with finite rate of innovation. IEEE Trans. Signal Process., 50(6):1417-1428, Jun. 2002. 